\title{
CONCEPTION OF PHOTO-INJECTORS FOR THE CTF3 EXPERIMENT
}

\author{
R. ROUX \\ Laboratoire de l'Accélérateur Linéaire, IN2P3-CNRS, Université de Paris-Sud, \\ B.P 34, 91898 Orsay, France
}

In the framework of the CLIC Test Facility (CTF3) under development at CERN, LALOrsay is responsible for the construction of two photo-injectors for two different linacs. One, dedicated for the so called "drive beam linac" must fulfil very demanding specifications. The RF gun has to provide a high quality beam composed of more than 2,000 bunches containing $2.33 \mathrm{nC}$ of charge each in one RF pulse. The model adopted is inspired from the CERN $3 \mathrm{GHz} 2-1 / 2$ cell type IV RF gun. We will summarize all the studies performed on the RF design and on the beam dynamics. The vacuum issue has been also carefully investigated. The constraints on the second photo-injector are less severe since it must be operated with one or 64 bunches of $0.5 \mathrm{nC}$ each for the so called "probe beam linac". It will also be a 2.5 cell gun at $3 \mathrm{GHz}$ but its design will be substantially different with respect to the former. This last project has only recently begun and therefore we will show only the preliminary results of the RF and of the beam dynamics simulations.

\section{Introduction}

For 5 years, the LAL is involved in the CLIC-Test-Facility 3 (CTF3) under construction at CERN. The goal of this accelerator is to test and valid the concepts of the multi-TeV linear collider CLIC, proposed by the CERN [1]. Schematically this accelerator is based on two linacs. One, the drive beam linac at $3 \mathrm{GHz}$, must provide a $150 \mathrm{MeV}$ electron beam with high charge which passing through resonant decelerating structures should produce high RF power at $30 \mathrm{Ghz}$. Then, this power is sent to the $30 \mathrm{GHz}$ travelling sections of the main linac where the electron beam is accelerated to the $\mathrm{TeV}$ energy for particle physics.

The drive beam linac of CTF3 was successfully operated with a thermionic gun built by the LAL [2,3] but it was decided to put a photo-injector in place of it because of the many advantages they have [4]. This project is a part of a Joint Research Activity, called PHIN, itself part of the Coordinated Accelerator 
Research in Europe ${ }^{*}$. Other work packages are defined in the context of an R\&D program on photo-cathodes and the construction of a custom laser to drive the photo-injector. In addition, the LAL is in charge of the construction of a second photo-injector for the probe beam linac which is used as the main linac in CLIC. The rest of the linac should be built by a French institute, the CEA-Saclay in collaboration with the CERN. The first part of this paper is dedicated to the RF design and the beam dynamic simulation of the PHIN photo-injector. In a second part, the photo-injector of the probe beam will be described mostly on the aspects which differ to the previous.

\section{The PHIN photo-injector}

LAL owns a good knowledge in the fabrication of photo-injectors. The first one home made, CANDELA with 1,5 cell, was dedicated to accelerator physics [5]. A second photo-injector for a small linac, ELYSE, according to a CERN type model, was built for radiolysis experiments in chemistry [6]. Now, for the CTF3 experiment, we decided to benefit from the CERN experience with CTF2 [7]. So, this gun is based on a previous proto-type CERN RF gun (2.5 cells, so called "type IV") which was built and tested with beam at CERN [8]. However, it needed further study in order to meet the specifications of the CTF3 drive beam linac (see table 1).

Table 1: Beam and RF gun parameters desired for CTF3.

\begin{tabular}{c|c} 
RF frequency $(\mathrm{GHZ})$ & 2.99855 \\
RF power $(\mathrm{MW})$ & 30 \\
Beam energy $(\mathrm{MeV})$ & $5-6$ \\
Beam current $(\mathrm{A})$ & 3.51 \\
Pulse train duration $(\mu \mathrm{s})$ & 1.548 \\
Bunch spacing $(\mathrm{ns})$ & 0.6666 \\
Charge/bunch $(\mathrm{nC})$ & 2.33 \\
Repetition rate $(\mathrm{Hz})$ & 5 \\
Bunch length, FWHM $(\mathrm{ps})$ & $<10$ \\
rms energy spread $(\%)$ & $<2$ \\
Normalized emittance $(\pi \mathrm{mmmrad})$ & $<25$ \\
Vacuum pressure $(\mathrm{mbar})$ & $<2.10^{-10}$
\end{tabular}

In the following, we show the results of the RF simulations performed with a 2-D electromagnetic code, SUPERFISH, and a 3-D code, HFSS, which

\footnotetext{
* We acknowledge the support of the European Community-Research Infrastructure Activity under the FP6 "Structuring the European Research Area" programme (CARE, contract number RII3-CT2003-506395)
} 
allowed us to obtain the main characteristics such as the cavity dimensions and the axial field distribution. Beam loading is also evaluated. Then simulations of the beam dynamics in the gun with the PARMELA code are summarised. In addition the vacuum aspects have been thoroughly investigated, as in previous experiments with RF photo-injectors at CERN the pressure has been found to increase dramatically at high levels of extracted charge $(\approx 1 \mu \mathrm{C})$.

\subsection{D RF Simulations}

The SUPERFISH simulations are used essentially to determine the physical dimensions of the photo-injector cavity. First, the main objectives are to check if the original design is compatible with the CTF3 parameters. We have studied, for example, the influence of the angle of the cathode wall as well as the shape of the iris. Of course, any change to the cavity must be compensated in order to keep the resonant frequency at the required value. The CERN design of the RF gun was optimised for higher charge by choosing the angle of the half-cell wall around the photo-cathode, to provide additional transverse focusing. According to electron beam dynamic simulations (see 1.3), this angle should be reduced to $3.4^{\circ}$ rather than the previous value $8^{\circ}$ in order to keep the energy spread within the desired limits.

Moreover, we have changed the shape of the iris from circular to elliptical to decrease the surface electric field and therefore it minimizes electrical breakdown and dark current levels [9]. Furthermore, we introduced a slight asymmetry in the walls of the cavities for mechanical reasons and to try to reduce multipactor hazards. The new design is shown schematically in Fig. 1.

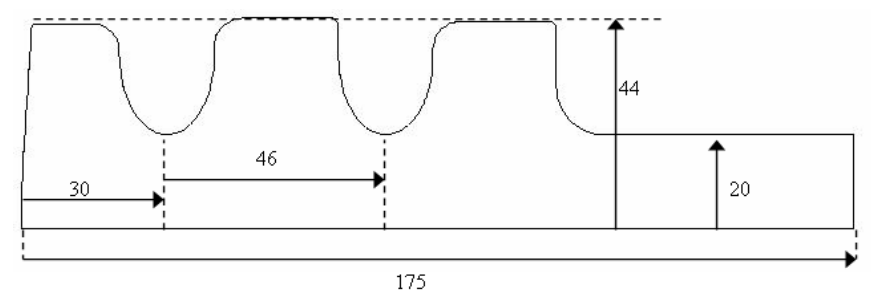

Figure 1: SUPERFISH design of the PHIN gun, lengths are in $\mathrm{mm}$.

The evidence of the efficiency of the elliptical iris for the reduction of the surface electric field with respect to the circular one is illustrated in Fig. 2. At first sight, the gain is not obvious since the average field is roughly the same in the two cases. But the peak electric field, one the irises, in the elliptical design is $15 \%$ lower with respect to the circular case. Now, the yield of the field emitted electrons by the cavity walls is strongly non linear with the electric field. 
Therefore the use of the elliptical iris should be helpful for the reduction of discharge problems due to field emission.

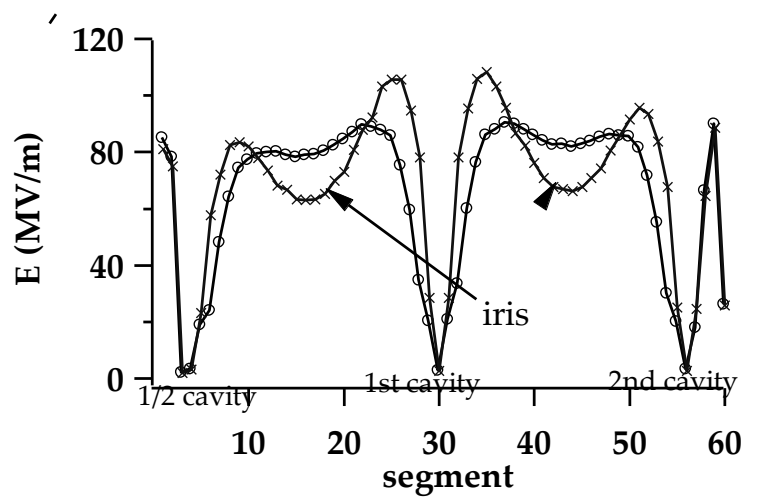

Figure 2: Surface electric field as a function of the SUPERFISH segment on the circumference of the model in figure 1. The surface electric field is zero in the mid-cells and maximum on the iris. The circles show the elliptical iris case and the crosses correspond to the circular iris case. The axial electric field is $85 \mathrm{MV} / \mathrm{m}$.

The parameters of this RF gun are shown in table 2. Finally, the main difficulty in designing the gun is to obtain good axial electric field "flatness" in every cell of the structure. This requirement is rather difficult to meet as the cells are strongly coupled and any change in the radius of one cell induces a variation of the electric field in every cell. The best adjustment we have obtained is shown in Fig. 3.

Table 2: RF photo-gun parameters
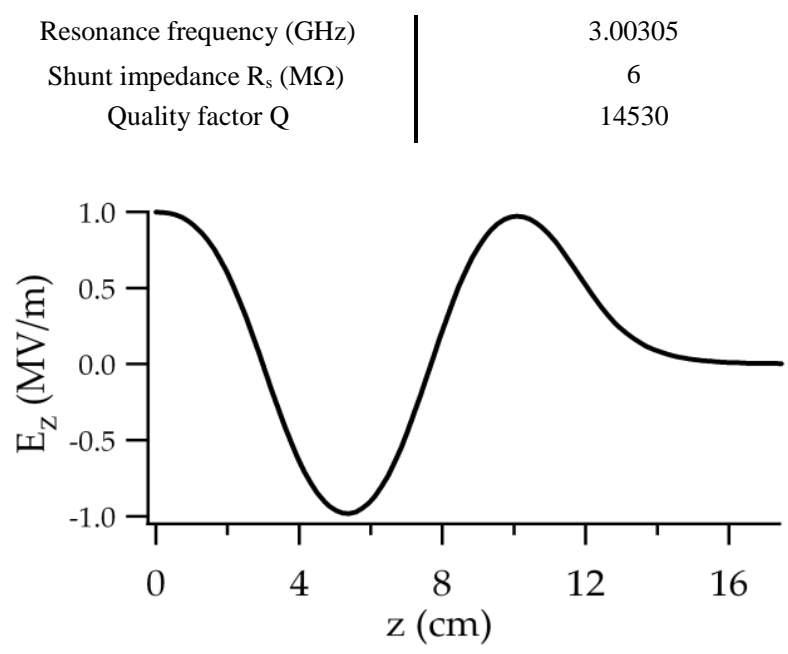

Figure 3: normalized axial electric field of the RF photo-gun. 


\subsection{Calculation of the Beam-Loading}

It is well known that the electron beam interacting with the impedance of the RF structures induces a beam loading voltage which can be detrimental for gun operation [10]. An important issue to consider is the HF matching of the photo-injector. The reflection factor depends on the coupling, $\beta$. In presence of the electron beam, the coupling is given by:

$\beta=\frac{\beta_{0}}{1+\frac{\mathrm{P}_{\text {beam }}}{\mathrm{P}_{\mathrm{RF}}}}$

where $\beta_{0}$ is the unloaded cavity coupling, $\mathrm{P}_{\mathrm{RF}}$ is the RF power dissipated into the cavity walls and $\mathrm{P}_{\text {beam }}=\mathrm{V} * \mathrm{I}$ is the power of the electron beam at the output of the RF gun.

For the nominal current and electric field, the beam energy is $5.5 \mathrm{MeV}$ hence $\mathrm{P}_{\text {beam }}$ is $19.3 \mathrm{MW}$. The necessary RF power is $9.6 \mathrm{MW}$; therefore, to get $\beta$ $=1$ and no reflection in presence of the beam, the RF gun must be over-coupled with $\beta_{0}=3$. It also means we need roughly $30 \mathrm{MW}$ of RF power to compensate for the electron beam consumption. Besides the matching, the beam-loading induces a supplementary energy spread over the train of bunches. The beam loading voltage evolves as cavity voltage induced by the RF input power. Both grow exponentially with the time constant $\tau=2 \mathrm{Q}_{\mathrm{L}} / \omega_{\mathrm{r}}$ where $\mathrm{Q}_{\mathrm{L}}$ and $\omega_{\mathrm{r}}$ are respectively, the cavity loaded quality factor and the resonant frequency. To avoid a decrease of the accelerating gradient over the train of bunches, one needs to inject the beam during the rise time of the electric field. In this way, the induced voltage is compensated by the increase of the RF input power resulting in a constant accelerating voltage. The time $t_{0}$ of injection is obtained from the evolution of the electric field due to the HF input power:

$$
\begin{aligned}
& \mathrm{E}(\mathrm{t})=\mathrm{E}_{\text {max }}\left(1-\exp \left(-\frac{\mathrm{t}}{\tau}\right)\right) \\
& \mathrm{t}_{0}=-\tau \ln \left(1-\frac{\mathrm{E}\left(\mathrm{t}_{0}\right)}{\mathrm{E}_{\text {max }}}\right)
\end{aligned}
$$

with $\mathrm{E}\left(\mathrm{t}_{0}\right)$, the accelerating peak electric field, e.g. $85 \mathrm{MV} / \mathrm{m}$ and $\mathrm{E}_{\max }$ the electric field which would be established corresponding to $30 \mathrm{MW}$ if no beam was injected. But without beam, the RF gun is not matched and the reflection factor is $25 \%$. So, only $22.5 \mathrm{MW}$ goes into the gun which gives $\mathrm{E}_{\max }=130$ $\mathrm{MV} / \mathrm{m}$. Using (2), one finds $\tau=0.385 \mu$ s and $\mathrm{t}_{0}=0.408 \mu \mathrm{s}$. 


\subsection{D RF Simulations}

The RF power is transmitted to the photo-gun via wave-guides and enters the gun through coupling holes whose aperture must be adjusted to, in principle, minimise the reflected power. In our case, as explained in the previous paragraph, we must design the gun to get a coupling factor, $\beta$, to be 2.9 which will allow the gun to be at critical coupling in the presence of a beam at the nominal current of 3.51 A. Moreover, for beam emittance preservation, the electric field must be kept symmetric around the axis. Consequently we decided to connect two couplers symmetrically with respect to the horizontal plane. The 3-D design is shown in Fig. 4.

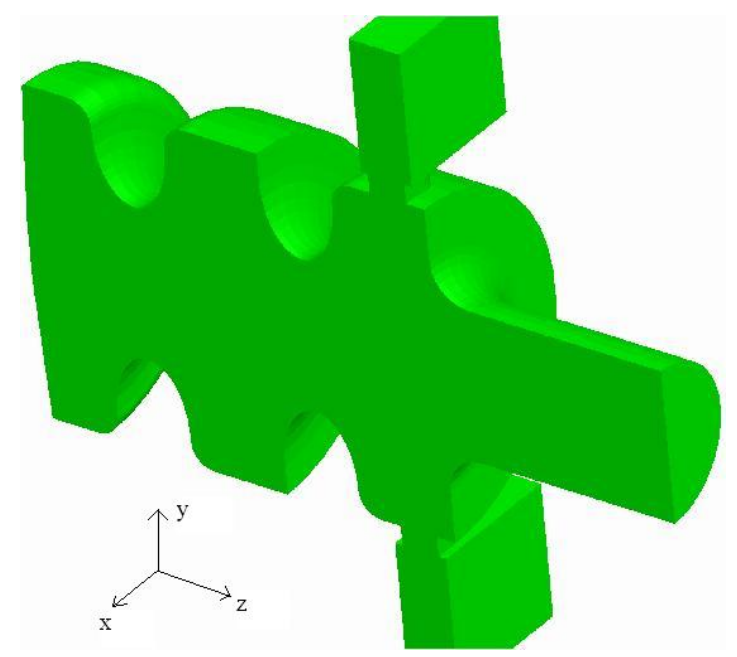

Figure 4: Half view of 3-D model of the RF photo-gun.

To leave enough space to install a solenoid around the gun it was decided to connect the couplers to the output cell and to use wave-guides whose inner height is $14 \mathrm{~mm}$ rather than the $3 \mathrm{GHz}$ standard $34 \mathrm{~mm}$. The shape of the coupling aperture is in the form of a racetrack and its dimensions are approximately $25 \mathrm{~mm} \times 10 \mathrm{~mm}$. This model was obtained after many simulations since three conditions need to be satisfied in simultaneously: the resonant frequency, the coupling and the field-flatness. For instance, according to the simulations it is necessary to have the radius of the last cell $1 \mathrm{~mm}$ smaller than in the second cell.

One last issue to check is the transverse symmetry of the electric field in the coupling cell. Thanks to the dual coupling, the field is symmetric vertically 
with respect to the $(\mathrm{x}, \mathrm{z})$ plane. But, inevitably, there is a difference of the field amplitude between the $\mathrm{x}$ and $\mathrm{y}$ direction. It adds a quadrupolar component to the acceleration in the transverse plane which leads to a degradation of the emittance. One solution to counteract this problem is to have a completely symmetrical coupling, namely a coaxial coupling with a hollow cylindrical antenna going into the RF gun along the axis of the cut-off tube. This scheme is now becoming standard equipment on the photo-injectors dedicated for free electron laser experiments striving to reach ultra-low emittance of $\sim 1 \mu \mathrm{mrad}$ [11]. But, in our case, we need a strong over-coupling which pushes the antenna, according to the simulations, deeply into the RF gun. The antenna would be practically at only $4 \mathrm{~mm}$ away from the iris between the middle cell and the coupling cell in an area of high electric field. In addition, the coaxial coupler has to support $30 \mathrm{MW}$ of $\mathrm{HF}$ power. We considered all these features would enhance the breakdown hazards, hence we gave up this option. Nevertheless, it is possible to improve the electric field symmetry in the case of the dual waveguide coupling with a special design of the cavity. It was proposed by $\mathrm{J}$. Haimson [12] to use a racetrack shape for the coupling cell in order to damp the asymmetry between both transverse directions (see drawing in figure 5a). Results of the HF simulations with this design are showed in figure 5b. First, it appeared that the field asymmetry is below $1 \%$ in the case of the usual cylindrical shape. But, between 0 and $6 \mathrm{~mm}$, where most of the particles are accelerated, the numerical noise, due to the finite number of tetrahedron, is dominating and makes difficult to analyse the results.
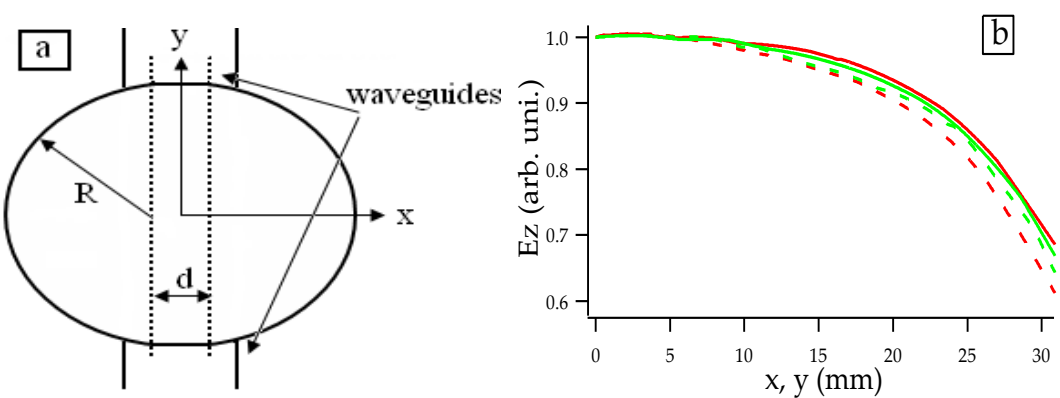

Figure 5: left, drawing of the section of the coupling cell; right, magnitude of the longitudinal electric field as a function of the distance in both transverse directions, red lines stand for the pure cylindrical cavity, green lines are for the racetrack cavity, plain lines represent the field along the $y$ axis while the dash lines is along the $\mathrm{x}$ axis.

The goal is to reduce as much as possible the relative difference of the electric magnitude in the area of interest. With the racetrack shape of the cavity, if one looks far away from the axis at $25 \mathrm{~mm}$, where the asymmetry is dominant with respect to the noise, the asymmetry is reduced by a factor 3 . Then, it is very 
likely that the field asymmetry is reduced in the same proportions in the space volume where the electron beam is present. The best reduction of the asymmetry, shown in figure 5 , is obtained with a very small value of the straight part of the racetrack, only $0.6 \mathrm{~mm}$ is necessary.

\subsection{Dynamics of the Electron Beam}

The simulations with PARMELA* presented in this section are based on the real longitudinal electric field calculated with SUPERFISH for the 2D model shown in figure 1. Unfortunately, it is not possible to extract the electric field of the HFSS model and to use it in PARMELA. Most of the simulations are done with 600 macro-particles for the study of the beam performances as a function of all parameters as the accelerating phase and gradient. Once the operation point is well defined, we will give beam performances with the maximum precision reachable with ten thousand particles. The results are often shown in the $\mathrm{x}$ direction because of the cylindrical symmetry and generally there are zero losses in the gun.

Several aspects of the beam dynamics have been investigated. First, calculations on the gun alone will be presented. Then, a study of the compensation of the beam emittance increase due to the space charge forces will be presented. The last simulations show the influence of the laser profile shape on the performances of the gun.

\subsubsection{The natural behavior}

The CERN design of the RF gun was optimised for higher charge, e.g. the choice of the angle of the wall around the photo-cathode. Therefore, we decided to study the influence of this parameter on the beam dynamics. The results are summarised in table 3.

Table 3: results of the simulations with PARMELA with 600 particles and the nominal current of CTF3 $(3.51 \mathrm{~A})$ as a function of the wall-angle around the photo-cathode. The widths are rms values and the rms emittance $\varepsilon_{\mathrm{T}}$ is normalised.

\begin{tabular}{l|lll} 
Wall angle & $0^{\circ}$ & $3.4^{\circ}$ & $8.3^{\circ}$ \\
\hline$\sigma_{\mathrm{x}}(\mathrm{mm})$ & 3.4 & 3.25 & 3.04 \\
$\varepsilon_{\mathrm{T}}(\pi \mathrm{mm}-\mathrm{mrad})$ & 20.6 & 20.7 & 22.3 \\
$\sigma_{\mathrm{z}}(\mathrm{mm})$ & 1.11 & 1.15 & 1.2 \\
$\mathrm{rms} \Delta \mathrm{E} / \mathrm{E}(\%)$ & 0.6 & 0.75 & 2.6
\end{tabular}

\footnotetext{
* PARMELA from Los Alamos National Laboratory, modified by B. Mouton at LAL.
} 
The case of an angle of $8.3^{\circ}$ is incompatible with the gun specifications and the case of $3.4^{\circ}$ seems less interesting with respect to a vertical wall. However, with a higher current, a small wall-angle is advantageous as it adds a focusing force which compensates the increase of the space charge forces.

So, as we want to maintain the possibility of operating at a higher current, we decided to adopt this small angle on the photo-cathode wall. Then we investigated the optimum operation point for the photo-injector as a function of the two HF parameters: the phase, $\phi$, and the magnitude of the electric field, E. As far as the phase is concerned, it was found that at $55^{\circ}$ we have the maximum energy gain and the minimum emittance. At $35^{\circ}$ the energy spread is minimized. We chose $\phi=35^{\circ}$ because it is more important to improve the energy spread than the little increase of energy gain at $55^{\circ}$ and the slight degradation of the emittance can be largely compensated as we will see in next sections. As for the choice of the electric field magnitude, simulations showed that the emittance could be reduced by $10 \%$ at $120 \mathrm{MV} / \mathrm{m}$ with respect to the nominal gradient, 85 $\mathrm{MV} / \mathrm{m}$. In addition the bunch length and the beam radius are smaller and the beam energy $40 \%$ bigger. However, because of the beam-loading issue, 120 $\mathrm{MV} / \mathrm{m}$ would require a power of $50 \mathrm{MW}$ which is beyond the limit of the available HF generator and breakdowns hazards would be enhanced. So, the operating gradient will be $85 \mathrm{MV} / \mathrm{m}$.

Results of the simulations for the gun model shown in Fig. 1 are illustrated in Fig. 6. The beam parameters at the output of the gun are summarised in table 4.

Table 4 : Beam parameters for a beam charge of $2.3 \mathrm{nC}$ and a Gaussian laser spot $\sigma_{\mathrm{r}}=1.4 \mathrm{~mm}(\mathrm{rms})$, $\sigma_{\mathrm{t}}=4 \mathrm{ps}$ and 1000 particles.

\begin{tabular}{c|c}
$\mathrm{E}(\mathrm{MeV})$ & 5.452 \\
$\varepsilon_{\mathrm{x}}(\pi \mathrm{mmmrad})$ & 19.6 \\
$\sigma_{\mathrm{x}}(\mathrm{mm})$ & 3.2 \\
$\sigma_{\mathrm{z}}(\mathrm{mm}, \mathrm{ps})$ & $1.07,3.56$ \\
$\mathrm{rms} \Delta \mathrm{E} / \mathrm{E}(\%)$ & 0.36
\end{tabular}

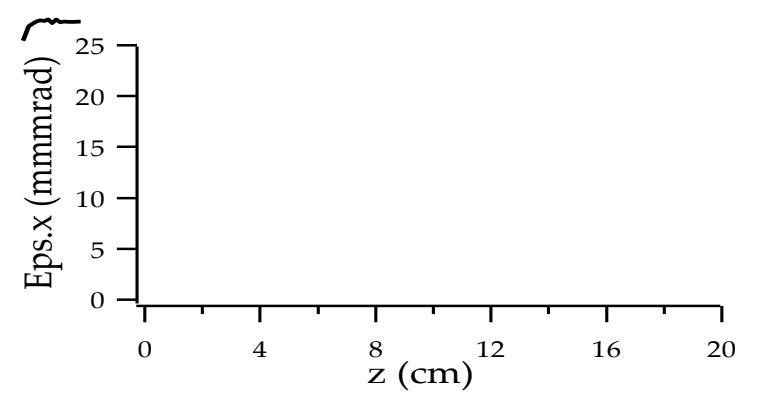

Figure 6: horizontal normalized emittance as a function of the longitudinal axis in the gun. 
The comparison of Table 4 and Table 1 shows the performances of the photo-injector fulfil the specifications of the drive beam of CTF3. The electron beam dynamic is dominated by space charge effect. In Fig. 6, most of the emittance growth occurs in the first half-cell when the beam energy is below 1 $\mathrm{MeV}$. Thanks to the magnetic focusing, it is possible somehow to reduce the emittance degradation.

\subsubsection{Compensation of the space charge forces}

Due to the space charge forces, the emittance grows linearly with the distance until the beam enters the accelerating section. At the output, the emittance is "frozen" because the space charge force is strongly damped at high energy. Therefore, to keep the emittance at the lowest possible value, it is necessary to use a transverse focusing between the output of the gun and the input of the section to compensate the transverse defocusing effect of the space charge. One possible technique, proposed by E. Carlsten [13], is the use of a magnetic lens. Simulations have been performed with a SLAC type section at the nominal current and $1.4 \mathrm{~mm}$ of laser spot size and with two coils for which the positions are variable (see figure 7). In all cases, thanks to a bucking coil, the magnetic field is less than one gauss on the photo-cathode. Besides, the fact that we used a SLAC section in the simulations has no influence on the conclusions which can be drawn from this study. For each couple of coil positions, we found the optimum value of the magnetic field for a good compensation of the emittance. Figure 7 shows that the biggest reduction of the emittance is obtained when the coils are placed near the photo-cathode. The only drawback is that it implies a stronger magnetic field, $0.25 \mathrm{~T}$ for the case where the coils are at $\mathrm{z}=2$ $\mathrm{cm}$ and $10 \mathrm{~cm}$.

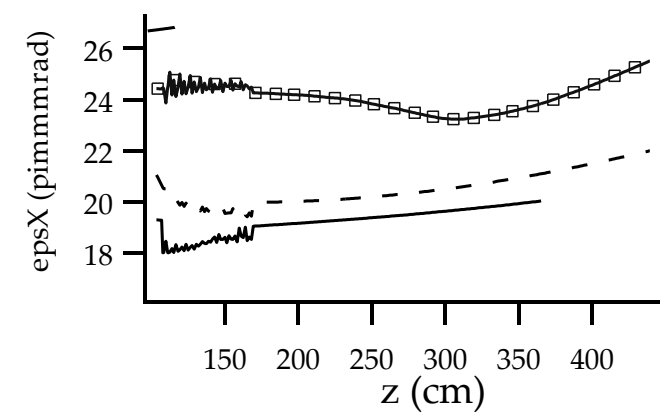

Figure 7: Emittance as a function of distance for several kinds of magnetic field. The SLAC section is located at $\mathrm{z}=107 \mathrm{~cm}$ and has a length of $63 \mathrm{~cm}$ and a gradient of $30 \mathrm{MV} / \mathrm{m}$. The position of the coils is variable; square points, coil at $\mathrm{z}=15 \mathrm{~cm}$ and $\mathrm{B}_{\mathrm{z}}=0.21 \mathrm{~T}$; dashed line, 2 coils at $\mathrm{z}=10 \mathrm{~cm}$ and $20 \mathrm{~cm}$ and $\mathrm{B}_{\mathrm{z}}=0.23 \mathrm{~T}$; plain line, 2 coils at $\mathrm{z}=2 \mathrm{~cm}$ and $10 \mathrm{~cm}$ and $\mathrm{B}_{\mathrm{z}}=0.25 \mathrm{~T}$. 
Space constraints around the gun are very stringent because of a heavy pumping system which will be detailed later. So, it was decided to install the bucking coil close to the photo-cathode plane and the focusing coil just downstream the waveguide coupled to the last cell. On this base, simulations of the coils have been performed with POISSON, the magnetic arrangement is showed in figure $8 \mathrm{a}$ and the corresponding beam dynamic in figure $8 \mathrm{~b}$.
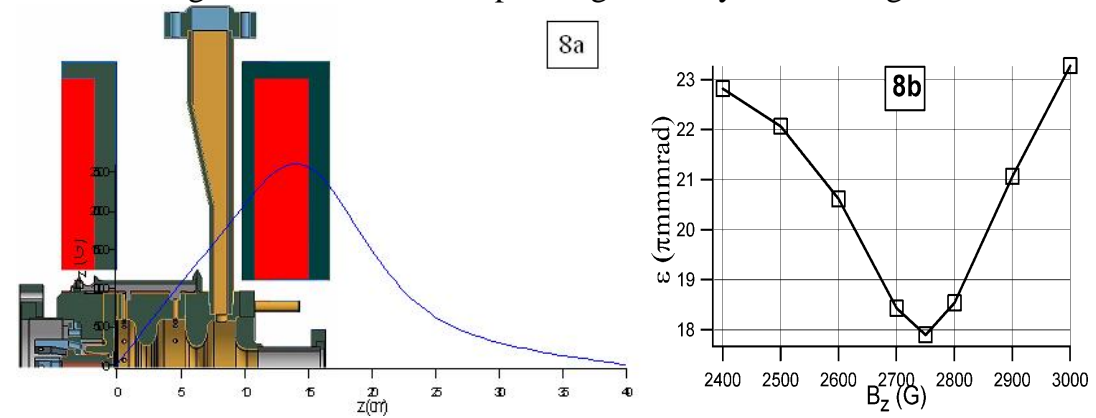

Figure 8: a, half section of the coils and magnetic field as a function of the longitudinal distance, grey blocks stands for iron and red is for copper; $b$, normalized emittance as a function of the peak magnetic field, PARMELA simulations with a Gaussian laser.

The dimensions of the coils which produce the required field lead to have 2 pancakes with 100 spires for the bucking coil and 4 pancakes with 208 spires for the focusing solenoid (for a square conductor of $6 \mathrm{~mm}$ side). The coils need a current of roughly 250 A from the power supply. Unfortunately, it was impossible to get the maximum of the magnetic field closer than the output of the gun. Nevertheless as it is illustrated in figure 8b, the compensation of the emittance growth due to space charge forces is quite effective and is obtained for $\mathrm{B}_{\mathrm{z}}=0.275 \mathrm{~T}$. The minimum rms normalized emittance is $18 \pi \mathrm{mmmrad}$ at a distance of roughly one meter from the photo-cathode and can be kept at this value if a traveling wave section is located at this coordinate.

\subsubsection{Influence of the laser profile shape}

The presence of the coils allowed one to cancel the emittance growth which would have occurred otherwise in the drift tube between the output of the gun and the entrance of the first section. To reduce more significantly the emittance, it is proposed to decrease the space charge forces thanks to a square profile of the laser pulse instead of a gaussian [14]. Indeed, the space charge effect is proportional to the electronic density and a more homogenous profile as a square profile should be less harmful to the emittance. In the PARMELA at our disposal, the only one available profile is the Gaussian. But it is still possible to simulate a square pulse thanks to a very large rms width and cutting the profile 
well inside the width. The simulation will not represent exactly the laser pulse because it is not possible to choose a rise time and a fall time. The principle of the simulation is illustrated in figure 9 .

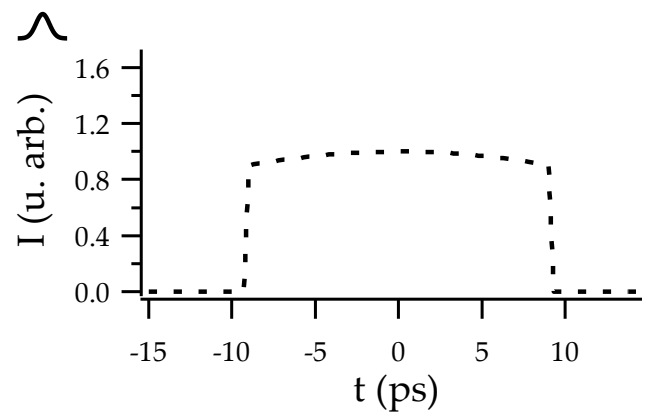

Figure 9: PARMELA simulations with different profiles of the laser temporal pulse; plain line, Gaussian shape with $\sigma_{\mathrm{t}}=4.2 \mathrm{ps}$; dashed line, Gaussian shape, with $\sigma_{\mathrm{t}}=20 \mathrm{ps}$ over the interval [-9, 9] ps, which is equivalent to a square laser profile.

During the simulations, it appeared that a very flat profile is not the best suited for the emittance. It induces beam losses in the gun. So, we looked for the optimum temporal and transverse widths to get the minimum emittance which is $11 \pi \mathrm{mmmrad}$, almost a factor 2 lower with respect to the case with a true Gaussian shape of the laser pulse. Then, we again investigated the minimization of the emittance with the coils with these square profiles and we found 9.6 $\pi$ mmmrad. The performances of the photo-injector are now optimised as a function of all parameters, a simulation with the maximum number of particles possible in our home PARMELA, 10000 particles, which lasted 10 hours, gave the final results summarised in table 5 .

\begin{tabular}{c|c}
$\mathrm{E}(\mathrm{MeV})$ & 5.452 \\
$\varepsilon_{\mathrm{x}, \mathrm{y}}(\pi \mathrm{mmmrad})$ & 5.6 \\
$\sigma_{\mathrm{x}, \mathrm{y}}(\mathrm{mm})$ & 1 \\
$\sigma_{\mathrm{z}}(\mathrm{mm}, \mathrm{ps})$ & $1.43,4.76$ \\
$\mathrm{rms} \Delta \mathrm{E} / \mathrm{E}(\%)$ & 1.3
\end{tabular}

Table 5 : Beam parameters for a beam charge of $2.3 \mathrm{nC}$ at the position of the minimum emittance ( $\mathrm{z}$ $=150 \mathrm{~cm}$ ) with the coils compensating the space charge effect, a square laser spot and 10000 particles.

Obviously, the energy of the beam did not change but the emittance, with a square 3D distribution of the laser pulse taking into account the coils and ten thousands of particles, is almost reduced by a factor 4 with respect to the initial results. This good performance is obtained to the cost of a small increase of the 
energy spread and bunch length because of the wider square distribution, but it is quite acceptable in regard to the specifications.

\subsection{Vacuum Calculations}

Vacuum considerations are a critical aspect of the project as the experiments performed at CERN showed it occurred an exponential growth of the pressure inside the gun as a function of the total extracted charge, whatever the bunch-train distribution is. Therefore, a thorough study of the residual pressure has been carried out to understand how we can attain a good vacuum in the RF photo-gun. The most relevant results are presented here but the overall analysis is given elsewhere [15]. A simplified model of the gun was used in the MONTE-CARLO based calculations. A pumping system is placed at $230 \mathrm{~mm}$ downstream of the gun and the out-gassing rate for the OFHC copper is assumed to be $5.10^{-11}$ mbar.1. $\mathrm{s}^{-1} \cdot \mathrm{cm}^{-2}\left(\mathrm{~N}_{2}\right.$ equivalent) for a standard cleaning and after roughly one hundred hours of pumping. Simulations showed that the pressure can not be lower than $2.10^{-9}$ mbar in the first half-cell because of the poor conductance of the photo-injector. In order to improve the quality of vacuum, it was decided to use NEG (Non Evaporative Getter) coating on the vacuum chamber at the output of the gun. In addition, we plan to install around the gun a cylindrical chamber which the inner surface is coated with NEG. It is connected to the vacuum of the gun thanks to 42 ducts drilled in the walls of the half-cell and the middle cell of the gun. A scheme of the design is showed in figure 10.

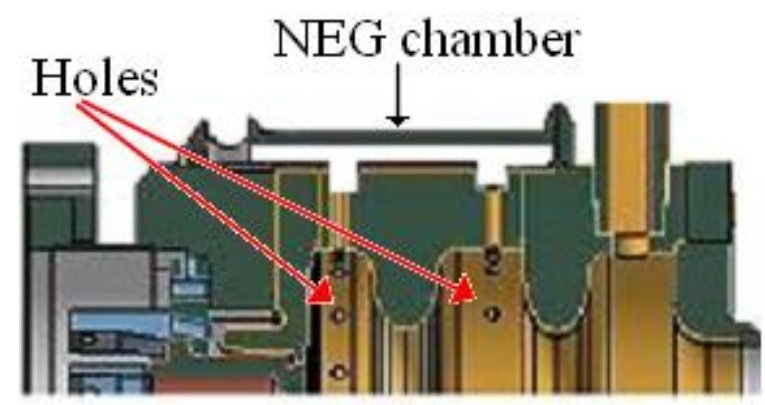

Figure 10: scheme of the NEG vacuum chamber around the photo-injector with the holes into the walls of the gun connecting both volumes.

Obviously a pre-pumping system is needed for this NEG chamber. In order to not to perturb the magnetic field, the ionic pumps must be exported outside the external radius of the coils. As a consequence, all the mechanical arrangement is very tight. Results of the simulations with this NEG pumping are illustrated in figure 11. 


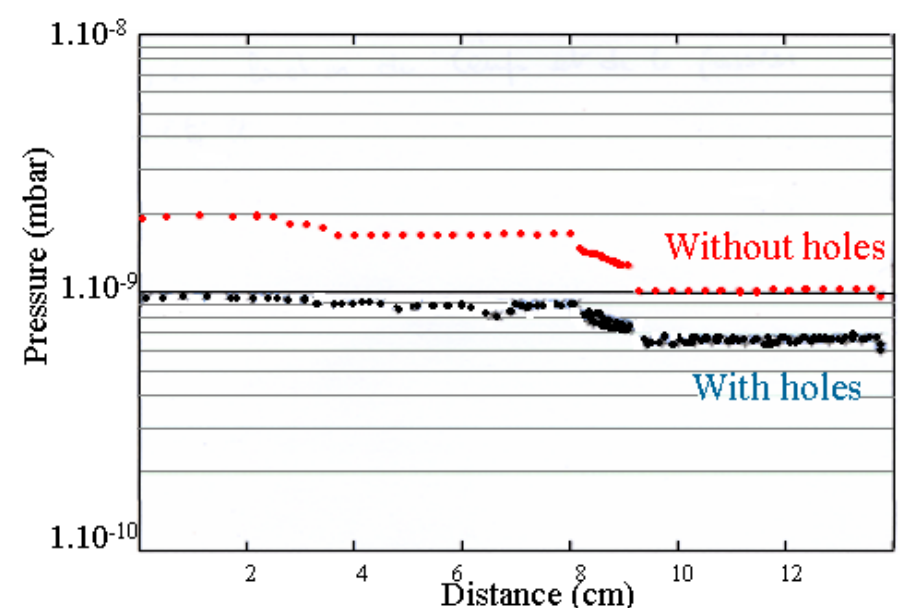

Figure 11: comparison of simulations of the residual pressure between the cases with and without NEG pumping.

The extra pumping coming from the NEG allows one to reduce the residual pressure by a factor 2 , achieving $10^{-9}$ mbar which is not satisfying yet. It is difficult to do better by lack of available space in the cell walls which hold also the cooling channels. In addition to the improvement of the static pressure, the NEG pumping can be helpful during the commissioning of the gun. As the pumping velocity is higher, time recovery of normal pressure after a sudden outgassing is significantly shorter (one minute less). But the only way to reach static pressure below $10^{-10}$ mbar is to lower the out-gassing rate of the copper. One solution is a high temperature bake $\left(800{ }^{\circ} \mathrm{C}\right)$ of the photo-injector. In this case, calculations predict a residual pressure of $10^{-11}$ mbar in the $1^{\text {st }}$ cell. Simulations with HFSS showed the impact of the presence of the 42 holes is negligible on the HF parameters ( $5 \%$ less on Q).

\section{The photo-injector of the probe beam}

The goal of the CTF3 accelerator is to test the technical challenges which arise in the CLIC project. Among them, the $30 \mathrm{GHz}$ accelerating sections must do the proof a beam can be propagated with a very high gradient, e.g. 150 $\mathrm{MV} / \mathrm{m}$, and with low losses. So, one linac in CTF3, the so called "probe beam linac", must provide such a beam. One agreement was found between CERN and two French institutes, LAL/IN2p3 from Orsay and DAPNIA/CEA-Saclay to build this linac. CERN provides advices and material (as the LIL accelerating sections), LAL is in charge of the construction of a photo-injector and DAPNIA should build the rest of the linac. In order to save time and money it was decided to take the PHIN gun as a start reference for the design of the probe beam photo- 
injector. Technical specifications required by CERN for the photo-injector are given in table 6.

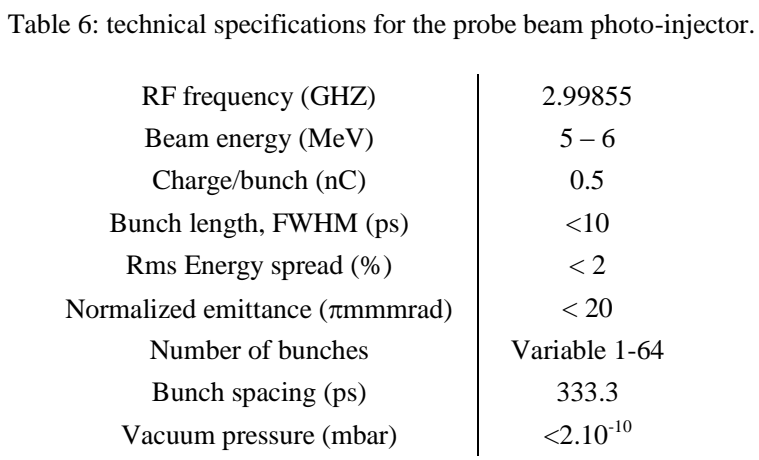

The biggest differences with respect to the requirements for the drive beam photo-injector are the charge per bunch, which is 4 times lower, and the number of bunches which can be several order of magnitude smaller. As a consequence, the tolerances on the design of photo-injector can be considerably relaxed. First, the charge per bunch and the duration of the bunch train ( $21 \mathrm{~ns}$ at maximum) are so small that the beamloading can be neglected. So there is no need to have overcoupling between the waveguides and the gun. The coupling apertures will be surely smaller therefore the dissymmetry of the electric field between the two transverse directions will be also reduced. It was decided not to use a racetrack shape of the coupling cell making the design simpler and cheaper for the machining. However this issue will be carefully checked. We also reduced the aperture of the irises down to $15 \mathrm{~mm}$ instead of $20 \mathrm{~m}$ because the electron beam radius should be significantly smaller with respect of that in the PHIN photoinjector. Finally the pumping system can be probably simplified. In the previous photo-injector a cylindrical NEG chamber is installed around the gun leading to a very complex mechanical assembling, since it was vital to improve the quality of vacuum (see 1.5). But in the case of the probe beam the total extracted charge, even in the worst case of 64 bunches, is two orders of magnitude lower than in the PHIN photo-injector. Therefore, it is probably safe to give up the idea to provide a NEG chamber in the probe beam photo-injector. We keep the option of a NEG coating on the downstream vacuum chamber of the gun because it requires no additional machining. HF design studies begun later than in the PHIN gun and are still under way. Preliminary results are given in table 7 for a design which is very similar to that of the drive beam photo-injector except the modifications above discussed. 
Table 7: results of the 2D HF simulations with SUPERFISH and beam dynamic simulations with PARMELA of the probe beam photo-injector.

$$
\begin{array}{c|c}
\mathrm{Q} & 14400 \\
\mathrm{R}_{\mathrm{s}}(\mathrm{M} \Omega) & 7.8 \\
\mathrm{E}_{\mathrm{acc}}(\mathrm{MV} / \mathrm{m}) & 80 \\
\text { HF Power }(\mathrm{MW}) & 5.2 \\
\text { Beam energy }(\mathrm{MeV}) & 5.35 \\
\text { Rms Energy spread }(\%) & 0.3 \\
\text { Normalized emittance }(\pi \mathrm{mmmrad}) & 6.5 \\
\text { Bunch length }(\mathrm{ps}), \mathrm{FWHM} & 8 \\
\text { Ream radius }(\mathrm{mm}), \mathrm{rms} & 2.3
\end{array}
$$

It is worth to notice that the required HF power is reduced by a factor 2 with respect of that in the PHIN photo-injector for a gradient which is slightly lower. So, we expect to have fewer troubles during the commissioning of the gun and the normal operation. According to the simulations all the electron beam parameters fulfil the specifications. The obtained emittance is for a Gaussian laser pulse, we could probably get a lower value with a square shape of the laser (see 1.4.3), close to the thermal emittance limit. Finally, it is foreseen to install coils on the photo-injector for the compensation of the emittance growth induced by the space charge forces.

\section{Conclusion}

The HF design and the electron beam dynamic of the drive beam photoinjector have been thoroughly investigated. The beam-loading induced by the beam is quite manageable and performances of the electron beam fulfill the CTF3 requirements. Since there is a tremendous total extracted charge which has to be produced, lot of efforts, as the envelop around the gun with NEG coating, have been carried out to improve the quality of the vacuum. Detailed technical drawings are finished and a prototype is being tested at LAL. Once HF measurements will have been confronted to the simulations the gun will be ordered to the manufacturer. So we hope the full equipped photo-injector will be installed and commissioned in the middle of 20006.

The photo-injector for the probe beam linac is less challenging. The HF design and the technical drawings are almost finished as we took benefit from the work achieved on the previous gun. Predictions of the electron beam performances are in agreement with the CTF3 requirements. The construction should take place during 2006 and the delivery to CERN one year after. 


\section{Acknowledgments}

The fabrication of two photo-injectors fully equipped does not only rely on the shoulders of the author but are the result of a team work. So, I would like to thank the drive beam photo-injector project leader, G. Bienvenu; B. Mouton for his considerable help in the simulations and computers; the engineers in Vacuum, B. Mercier and C. Prevost; the mechanical designers, J. Prevost and M. Desmons and a newcomer but enthusiastic in HF simulations, J. Brossard. I also thank T. Garvey who gave me the opportunity to present this work and A. Variola for his suggestions of the paper.

\section{References}

[1] H. H. Braun et al, CERN yellow report, CERN-99-06 (1999).

[2] R. Corsini et al, proc. of the $9^{\text {th }}$ Eur. Part. Acc. Conf. EPAC 2004, Lucerne, Switzerland, 05-09 Jul. 2004, p. 39.

[3] G. Bienvenu et al, proc. of the $8^{\text {th }}$ Eur. Part. Acc. Conf. EPAC 2002, Paris, France, 03-07 Jun. 2002, p. 1753.

[4] H. H. Braun et al, proc. of the Part. Acc. Conf., PAC 2001, Chicago, U. S. A., June 18-22 2001, p. 39.

[5] C. Travier et al, Nucl. Inst. and Methods, Vol A 393, pp451-454, 1997.

[6] T. Garvey et al, proc. of the 8th Eur. Part. Acc. Conf. EPAC2002, Paris, pp $254-256,2002$.

[7] H. H. Braun, $8^{\text {th }}$ CLIC/CTF3 meeting, Sept.2003, CERN, http://ctf3.home.cern.ch/ctf3/New collab meet.htm

[8] R. Bossart, H. Braun, M. Dehler, J.-C. Godot, Nucl. Inst. and Methods, Vol A 375, ABS7-ABS8, 1996.

[9] J. Luiten et al, proc. of the 9th Eur. Part. Acc. Conf. EPAC2004, Lucerne, Switzerland, p. 725, 2004.

[10] D. Boussard, Cern Accelerator Courses, $5^{\text {th }}$ Advanced Physics Course, Rhodes, Greece 1993, p. 415 (1995).

[11] B. Dwersteg, K. Flötmann, J. Sekutowicz, Ch. Stolzenburg, Nucl. Instr. Meth. in Phys. Research A 393 (1997), 93.

[12] J. Haimson, B. Mecklenburg and E. L. Wright, proceedings of the Advanced Accelerator Concepts conference 1998, AIP conference proceedings 398, pp. 898-911.

[13] B. E. Carlsten, Nucl. Instr. Meth. in Phys. Research A 285 (1989), 313.

[14] F. Zhou et al, proc. of the 8th Eur. Part. Acc. Conf. EPAC2002, Paris, p. 275, 2002.

[15] B. Mercier, C. Prevost, R. Roux, "Presentation of the vacuum simulations on the photo-injector for the CLIC Test Facility", to be submitted to Vacuum. 